\title{
3 parton production at DIS at small $x$
}

\author{
Martin Hentschinski ${ }^{1, \star}$ \\ ${ }^{1}$ Departamento de Actuaria, Física y Matemáticas, Universidad de las Americas Puebla, Santa Catarina \\ Martir, 72820, Puebla, Mexico
}

\begin{abstract}
We use the spinor helicity formalism to calculate the cross section for production of three partons of a given polarization in Deep Inelastic Scattering (DIS) off proton and nucleus targets at small Bjorken $\mathrm{x}$. The target proton or nucleus is treated as a classical color field (shock wave) from which the produced partons scatter multiple times. The resulting expressions are used to study azimuthal angular correlations between produced partons in order to probe the gluon structure of the target hadron or nucleus as well as to study energy loss in DIS reactions.
\end{abstract}

\section{Introduction}

The perturbative Regge limit of Quantum Chromodynamics (QCD) - which is characterized by the presence of a hard scale $Q^{2}$ far bigger than the QCD confinement scale and high center of mass energy $s$, such that $x=Q^{2} / s \ll 1-$ continues to provide challenges to those who wish to explore it. While Balitsky-Fadin-Kuraev-Lipatov (BFKL) evolution [1] is known to yield the correct perturbative high energy evolution equation, if the full resummation program is fully perturbative, there exist various theoretical arguments, which suggests that at some - yet unknown - value of energy, non-linear effects will complement BFKL evolution and tame the observed (and by BFKL predicted) rise of the perturbative gluon and bring it finally to hold. A dynamical mechanism which achieves such perturbative unitarity of QCD cross-sections at low $x$ is known under the term 'gluon saturation' and has been originally proposed by Gribov, Levin and Ryskin [2]. The framework of the Color Glass Condensate (CGC) provides on the other hand an effective action approach to gluon saturation [3]. Within this framework, the high energy hadron or nucleus is described as a a weakly-coupled yet non-perturbative system of gluons with high occupancy number, characterized by a semi-hard scale $Q_{s}$, called the saturation scale. As a result one obtains a non-linear generalization of the BFKL evolution equation [4-6], which includes multiple-scattering effects; the latter provide the necessary slow down and saturation of the growth with energy, see [7] and references therein

While the above mentioned non-linear dynamics is of high interest and many efforts are dedicated towards its exploration, one finds at the same time that at current collider energies, linear low $x$ evolution provides a pretty good description of data, see for instance the study [8] of exclusive vector meson production, where the energy dependence results directly of the NLO BFKL fit of [9]. To reveal saturation effects (or rather their onset) at current energies, it is therefore necessary to study specially

^e-mail: martin.hentschinski@udlap.mx 
designed observables, which provide increased sensitivity to the presence of high gluon densities. In the following we give some details on a calculation of such an observables as well as a first numerical simulation. The hope is that this observable will provide deeper inside into the question, whether we already experience the on-set of non-linear corrections or not. For more details we refer the interested reader to the original publication $[12,13]$.

\section{3 parton production in DIS at low $x$}

Since the CGC-framework assumes high gluon densities in the probed target, the gluonic field of the hadron/nucleus latter can be no longer treated perturbatively. The resulting formalism represents therefore the DIS scattering process in the low $x$ limit as the scattering of the virtual photon on a strong background color field; this field then essentially represents the target, together with a suitable averaging procedure over field configurations. Defining light-cone vectors $n, \bar{n}$ through the four momenta of virtual photon and target, i.e. $n \sim p$ and $n \cdot \bar{n}=1$ with $v^{-} \equiv n \cdot v, v^{+} \equiv \bar{n} \cdot v$ for a generic four vector $v$, theory calculations are performed using quark and gluon propagators in the presence of the background field $A^{+, c}$. One finds for the propagators

$$
\begin{gathered}
\tilde{S}_{F}(p, q)=(2 \pi)^{d} \delta^{(d)}(p-q) \tilde{S}_{F}^{(0)}(p)+\tilde{S}_{F}^{(0)}(p) \tau_{f}(p, q) \tilde{S}_{F}^{(0)}(q), \\
\tilde{G}_{\mu \nu}(p, q)=(2 \pi)^{d} \delta^{(d)}(p-q) \tilde{G}_{\mu \nu}^{(0)}(p)+\tilde{G}_{\mu \alpha}^{(0)}(p) \tau_{g}(p, q) \tilde{G}_{\alpha \nu}^{(0)}(q),
\end{gathered}
$$

with the free propagators

$$
\tilde{S}_{F}^{(0)}(p)=\frac{i \not p+m}{p^{2}-m^{2}+i 0}, \quad \tilde{G}_{\mu \nu}^{(0)}(p)=\frac{i d_{\mu \nu}(p)}{p^{2}+i 0}, \quad d_{\mu v}(p)=-g_{\mu v}+\frac{n_{\mu}^{-} p_{v}+p_{\mu} n_{v}^{-}}{n^{-} \cdot p},
$$

and the interaction terms

$$
\begin{aligned}
& \tau_{f}(p, q)=2 \pi \delta\left(p^{-}-q^{-}\right) h^{-} \int d^{d-2} z e^{-i z \cdot(p-q)}\left\{\theta\left(p^{-}\right)[V(z)-1]-\theta\left(-p^{-}\right)\left[V^{\dagger}(z)-1\right]\right\} \\
& \tau_{g}(p, q)=-2 \pi \delta\left(p^{-}-q^{-}\right) 2 p^{-} \int d^{d-2} z e^{-i z \cdot(p-q)} \cdot\left\{\theta\left(p^{-}\right)[U(z)-1]-\theta\left(-p^{-}\right)\left[U^{\dagger}(z)-1\right]\right\} .
\end{aligned}
$$

The latter contain Wilson lines in the fundamental $(V)$ and adjoint $(U)$ representation which resum the interaction with the background field with

$$
V(z) \equiv V_{i j}(z) \equiv \mathrm{P} \exp i g \int_{-\infty}^{\infty} d x^{-} A^{+, c}\left(x^{-}, z\right) t^{c}
$$

and $U^{b a}\left(z_{t}\right)=2 \operatorname{tr}\left(t^{a} V\left(z_{t}\right) t^{b} V^{\dagger}\left(z_{t}\right)\right) ; \mathrm{P}$ denotes path ordering of the gluon field in the exponent while $t^{c}$ is a $\mathrm{SU}\left(N_{c}\right)$ generator in the fundamental representation.

A well known possibility to increase gluon densities and therefore the sensitivity to saturation is to consider furthermore scattering on large nuclei. In such a nuclear environment, gluon densities are expected to be enhanced by a factor $A^{\frac{1}{3}}$, with $A$ the mass number of the nucleon. This sensitivity to high density effects is then furthermore enhanced, if one considers exclusive final states: while for inclusive observables the presence of high gluon densities leads mainly to a modification of the $x$ dependence of gluon densities [11], exclusive production processes can provide matrix elements which differ for high gluon densities from their low density counter parts. They are therefore more 


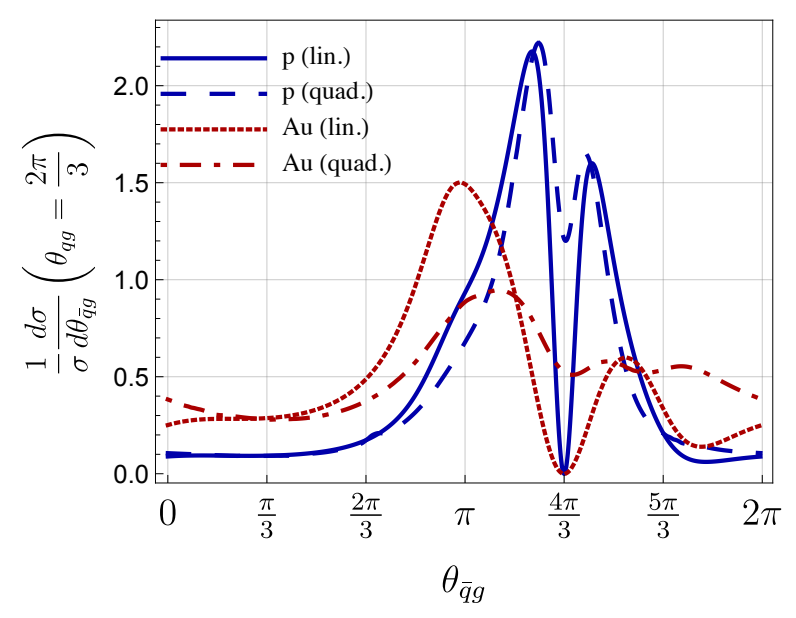

Figure 1. In the Mercedes-Benz star configuration, all transverse momenta have equal absolute value, with relative angle $\Delta=2 \pi / 3$. We show the normalized cross-section for three parton production in the presence of large gluon densities against $\Delta \theta_{\bar{q} g}$ with $\Delta_{q g}=2 \pi / 3$ for proton and gold up to linear and quadratic order in $N^{(2)}$. For the study the photon momentum fractions of the final state particles have been fixed to $z_{1}=z_{2}=0.2, z_{3}=0.6$ while $\left|\boldsymbol{p}_{1}\right|=\left|\boldsymbol{p}_{2}\right|=\left|\boldsymbol{p}_{3}\right|=2 \mathrm{GeV}$ and $Q^{2}=9 \mathrm{GeV}^{2}$ (plot originally published in [12]).

sensitive to effects related to high gluon densities and provide a tool for their exploration. In the following we study the process

$$
\gamma^{*}(q)+\operatorname{target}(p) \rightarrow q\left(p_{1}\right)+\bar{q}\left(p_{2}\right)+g\left(p_{3}\right)+X,
$$

where we refer for details to $[12,13]$. This observable has the big advantage that it depends on the gluon distribution in the target in a highly non-linear way and therefore gives hope to reveal the presence of such non-linear effects. The results of a first numerical study are shown in Fig. 1. With the differential cross-section for the process Eq. (5) expressed in terms of dipole and quadrupole operators of Wilson lines Eq. (4),

$$
S_{\left(x_{1} x_{2}\right)}^{(2)} \equiv \frac{1}{N_{c}} \operatorname{tr}\left[V\left(x_{1}\right) V^{\dagger}\left(x_{2}\right)\right], \quad S_{\left(x_{1} x_{2} x_{3} x_{4}\right)}^{(4)} \equiv \frac{1}{N_{c}} \operatorname{tr}\left[V\left(x_{1}\right) V^{\dagger}\left(x_{2}\right) V\left(x_{3}\right) V^{\dagger}\left(x_{4}\right)\right],
$$

this study uses the large $N_{c}$ and Gaussian approximation [14] to express the quadrupole in terms of the dipole operator. For the dipole profile itself a model motivated by recent rcBK$^{1}$ fits to HERA data [15] has been used,

$$
\begin{aligned}
& S_{\left(\boldsymbol{x}_{1} \boldsymbol{x}_{2}\right)}^{(2)}=\int d^{2} \boldsymbol{l} e^{-i \boldsymbol{l} \cdot \boldsymbol{x}_{12}} \Phi\left(\boldsymbol{l}^{2}\right)=2\left(\frac{Q_{0}\left|\boldsymbol{x}_{12}\right|}{2}\right)^{\rho-1} \frac{K_{\rho-1}\left(Q_{0}\left|\boldsymbol{x}_{12}\right|\right)}{\Gamma(\rho-1)} \\
& \text { where } \quad \Phi\left(\boldsymbol{l}^{2}\right)=\frac{\rho-1}{Q_{0}^{2} \pi}\left(\frac{Q_{0}^{2}}{Q_{0}^{2}+\boldsymbol{l}^{2}}\right)^{\rho},
\end{aligned}
$$

and $Q_{0}$ is a scale proportional to the saturation scale. To justify the dilute limit associated with the Gaussian approximation, we study the cross-section at large photon virtuality $Q^{2}=9 \mathrm{GeV}^{2}$

\footnotetext{
${ }^{1}$ The running coupling Balitsky-Kovchegov equation (rcBK) provides a non-linear generalization of the BFKL equation as discussed above, see [5, 15] for details
} 
and expand the differential cross-section up to quadratic order in $N^{(2)}=1-S^{(2)}$. We further use $\rho=2.3$ and $Q_{0}^{\text {proton }}=0.69 \mathrm{GeV}$, as motivated by inclusive DIS fits at $x=0.2 \times 10^{-3}$. Since we want to search for effects due to large gluon densities, we further simulate scattering on a gold nucleus through the re-scaling $Q_{0}^{\mathrm{Au}}=A^{1 / 6} \cdot Q_{0}^{\mathrm{proton}}=1.67 \mathrm{GeV}$. At linear order in $N^{(2)}$, the cross-section is proportional to the Fourier transform of the dipole, $\Phi\left(\left(\boldsymbol{p}_{1}+\boldsymbol{p}_{2}+\boldsymbol{p}_{3}\right)^{2}\right)$ and therefore gives direct access to the gluon distribution in the target. At this order the cross-section is - up to the detailed shape and $x$-dependence of the dipole factor Eq. (7) - identical to a corresponding BFKL calculation. The configuration dominant in the 'collinear' limit corresponds then to the vanishing of the sum of transverse momenta $\boldsymbol{p}_{1}+\boldsymbol{p}_{2}+\boldsymbol{p}_{3}=0$, i.e. the transverse momentum transfer between projectile and target is zero. To study deviations from this configuration we first chose $\left|\boldsymbol{p}_{1}\right|=\left|\boldsymbol{p}_{2}\right|=\left|\boldsymbol{p}_{3}\right|$. For this choice the 'collinear' limit $\boldsymbol{p}_{1}+\boldsymbol{p}_{2}+\boldsymbol{p}_{3}=0$ is then given by the Mercedes-Benz star configuration, see Fig. 1. We observe vanishing of the partonic cross section at these 'collinear' configurations, Fig. 1, with a strong double peak. This vanishing is explained by the vanishing of the partonic matrix element at leading order in $N^{(2)}$ for zero momentum transfer between projectile and target, which itself is expected from gauge invariance. For a complete study at hadronic level, we expect the double peak to turn into a single peak. While in the case of the proton we find (besides the double-peak structure) mainly a smearing of the collinear peak at $\Delta_{\bar{q} g}=4 \pi / 3$, the effect of a larger gluon saturation scale for a gold nucleus can be clearly seen: while the shape of the linear gold curve is similar to the proton curve, it is wider and depleted with respect to the former. Including further quadratic terms in $N^{(2)}$, we start to probe effects due to high gluon densities at the level of the matrix element beyond the mere modification of Eq. (7) and beyond the corresponding BFKL matrix element. In the case of the proton, these corrections are small, as expected for $x=0.2 \cdot 10^{-3}$ and a relatively large photon virtuality $Q^{2}=9 \mathrm{GeV}^{2}$. For a highly saturated gold nucleus, they are on the other hand sizable. While this apparently puts doubts on the reliability of the current expansion up to quadratic order in $N^{(2)}$, it clearly demonstrates the sensitivity of this processes to high and saturated gluon densities and identifies it as suitable tool in searching for such effects already at currently accessible collider energies.

\section{Conclusions}

In this contribution, we provided a short overview on the use of the 3 parton production process in Deep Inelastic Scattering to pin down the presence of high density effects. Those effects in turn would then allow a further exploration of the dynamics associated with gluon saturation.

\section{Acknowledgments}

I am grateful for support by the symposium organizers and the Red Mexicana Científica y Tecnológica para ALICE LHC.

\section{References}

[1] L. N. Lipatov, Sov. J. Nucl. Phys. 23 (1976) 338, E. A. Kuraev, L. N. Lipatov, V. S. Fadin, Phys. Lett. B 60 (1975) 50, Sov. Phys. JETP 44 (1976) 443, Sov. Phys. JETP 45 (1977) 199. I. I. Balitsky, L. N. Lipatov, Sov. J. Nucl. Phys. 28 (1978) 822; V. S. Fadin, L. N. Lipatov, Phys. Lett. B 429 (1998) 127, M. Ciafaloni, G. Camici, Phys. Lett. B 430 (1998) 349.

[2] L. V. Gribov, E. M. Levin and M. G. Ryskin, Phys. Rept. 100, 1 (1983). 
[3] L. D. McLerran and R. Venugopalan, Phys. Rev. D 49, 2233 (1994), Phys. Rev. D 49, 3352 (1994).

[4] J. Jalilian-Marian, A. Kovner, L. D. McLerran and H. Weigert, Phys. Rev. D 55, 5414 (1997); J. Jalilian-Marian, A. Kovner, A. Leonidov and H. Weigert, Nucl. Phys. B 504, 415 (1997), Phys. Rev. D 59, 014014 (1999), Phys. Rev. D 59, 014015 (1999), Phys. Rev. D 59, 034007 (1999), A. Kovner, J. G. Milhano and H. Weigert, Phys. Rev. D 62, 114005 (2000); A. Kovner and J. G. Milhano, Phys. Rev. D 61, 014012 (2000); E. Iancu, A. Leonidov and L. D. McLerran, Nucl. Phys. A 692, 583 (2001), Phys. Lett. B 510, 133 (2001); E. Ferreiro, E. Iancu, A. Leonidov and L. McLerran, Nucl. Phys. A 703, 489 (2002); H. Weigert, Nucl. Phys. A703, 823-860 (2002); J. -P. Blaizot, E. Iancu, H. Weigert, Nucl. Phys. A713, 441-469 (2003).

[5] I. Balitsky, Nucl. Phys. B 463, 99 (1996); Y. V. Kovchegov, Phys. Rev. D 60, 034008 (1999), Phys. Rev. D 61, 074018 (2000).

[6] Y. V. Kovchegov and E. Levin, Nucl. Phys. B 577, 221 (2000) [hep-ph/9911523]; M. Hentschinski, H. Weigert and A. Schafer, Phys. Rev. D 73, 051501 (2006) [hep-ph/0509272]; E. Levin and A. Prygarin, Phys. Rev. C 78, 065202 (2008) [arXiv:0804.4747 [hep-ph]].

[7] E. Iancu and R. Venugopalan, In *Hwa, R.C. (ed.) et al.: Quark gluon plasma* 249-3363; H. Weigert, Prog. Part. Nucl. Phys. 55, 461 (2005); J. Jalilian-Marian, Y. V. Kovchegov, Prog. Part. Nucl. Phys. 56, 104-231 (2006); F. Gelis, E. Iancu, J. Jalilian-Marian, R. Venugopalan, Ann. Rev. Nucl. Part. Sci. 60, 463-489 (2010); J. L. Albacete and C. Marquet, Prog. Part. Nucl. Phys. 76, 1 (2014).

[8] I. Bautista, A. Fernandez Tellez and M. Hentschinski, Phys. Rev. D 94 (2016) no.5, 054002 doi:10.1103/PhysRevD.94.054002 [arXiv:1607.05203 [hep-ph]].

[9] M. Hentschinski, A. Sabio Vera and C. Salas, Phys. Rev. Lett. 110 (2013) no.4, 041601 [arXiv:1209.1353 [hep-ph]].

[10] M. Hentschinski, A. Sabio Vera and C. Salas, Phys. Rev. D 87 (2013) no.7, 076005 [arXiv:1301.5283 [hep-ph]].

[11] J. Bartels and K. Kutak, Eur. Phys. J. C 53, 533 (2008) [arXiv:0710.3060 [hep-ph]]; L. Motyka and M. Sadzikowski, Acta Phys. Polon. B 45, no. 11, 2079 (2014) [arXiv:1411.7774 [hep-ph]].

[12] A. Ayala, M. Hentschinski, J. Jalilian-Marian and M. E. Tejeda-Yeomans, arXiv:1604.08526 [hep-ph].

[13] A. Ayala, M. Hentschinski, J. Jalilian-Marian and M. E. Tejeda-Yeomans, Nucl. Phys. B 920 (2017) 232 doi:10.1016/j.nuclphysb.2017.03.028 [arXiv:1701.07143 [hep-ph]].

[14] F. Dominguez, C. Marquet, B. -W. Xiao and F. Yuan, Phys. Rev. D 83, 105005 (2011).

[15] J. L. Albacete, N. Armesto, J. G. Milhano, P. Quiroga-Arias and C. A. Salgado, Eur. Phys. J. C 71, 1705 (2011). 